\title{
Risk factors for impaired health-related quality of life in a cohort of pediatric patients with inborn metabolic diseases
}

\author{
Sandy Siegert ${ }^{1} \cdot$ Anne Roscher $^{1} \cdot$ Dorothea Moeslinger $^{1} \cdot$ Vassiliki Konstantopoulou $^{1} \cdot$ Marion Herle $^{1} \mathbb{D}$
}

Received: 15 July 2021 / Revised: 17 October 2021 / Accepted: 18 October 2021 / Published online: 31 October 2021

(c) The Author(s) 2021

\begin{abstract}
In the last decade, health-related quality of life (HrQoL) has become an increasingly important outcome parameter in children and adolescents with chronic health conditions; among them are pediatric patients with inborn metabolic diseases (IMDs). Hence, knowledge on this topic is increasing, but findings on non-medical influences on the HrQoL of IMD patients are still scarce. In the present study, we retrospectively evaluated the self-reported generic HrQoL of a cohort of pediatric patients (ages 7 to 17 years) with diverse IMDs $(n=204)$ and explored associations between HrQoL and psychosocial and medical characteristics of the patients. We aimed to identify risk factors for impaired HrQoL to improve and tailor support for the patients and economize resources. Generic HrQoL was assessed with the KINDL-R questionnaire. We compared the HrQoL scores to published German normative data and analyzed the impact of demographic variables and intellectual and psychosocial functioning on the HrQoL. Moreover, we examined the influence of the diagnostic category and the health impairment (as judged by the physicians) on our patients' HrQoL. Overall, the HrQoL of the adolescent patients was comparable to the HrQoL of the norm group. Disorders of intellectual development, impaired psychosocial functioning, and a severe health impairment were associated with lower HrQoL scores.

Conclusion: We recommend evaluating these factors in children and adolescents with IMDs to identify patients at risk for impaired HrQoL.

\section{What is Known:}

- Studies on HrQoL in pediatric patients with IMDs mainly focused on subgroups with specific diagnoses and found normal HrQoL in some of those subgroups.

- In healthy children and adolescents as well as in pediatric patients with various chronic diseases, associations between psychosocial factors and HrQoL are well known.

What is New:

- Impaired psychosocial functioning, disorders of intellectual development, and a significant disease and/or treatment burden are risk factors for impaired $\mathrm{HrQoL}$ in pediatric patients with IMDs.

- Evaluating these factors in children and adolescents with IMDs can help identify patients and families in need of enhanced psychological support.
\end{abstract}

Keywords Health-related quality of life $\cdot$ Inborn metabolic diseases $\cdot$ Inborn errors of metabolism $\cdot$ Risk factors $\cdot$ Children

\section{Abbreviations}

AID 2

Adaptive Intelligence Diagnosticum 2

ANOVA

Analysis of variance

Communicated by Peter de Winter

Marion Herle

marion.herle@meduniwien.ac.at

1 Department of Pediatrics and Adolescent Medicine, Medical University of Vienna, Währinger Gürtel 18-20, 1090 Vienna, Austria
HrQoL Health-related quality of life

IQ Intelligence quotient

IMD Inborn metabolic disease

K-ABC Kaufman Assessment Battery for Children

PKU Phenylketonuria

Total HrQoL KINDL total HrQoL score

SDQ Strengths and Difficulties Questionnaire

SSIEM Society for the Study of Inborn Errors of Metabolism 


\section{Introduction}

Inborn metabolic diseases (IMDs) represent a large, heterogeneous group of genetic disorders that lead to a dysfunctional metabolism with observable effects already in early childhood. A classification of inherited metabolic disorders is provided by the Society for the Study of Inborn Errors of Metabolism (SSIEM) [1]. Due to these diseases' heterogeneity, patients exhibit mild to severe symptoms and are faced with different and sometimes challenging therapeutic options. Despite therapy, some of these conditions still lead to developmental disturbances, disorders of intellectual development, organ damage, physical impairment, and a shortened life expectancy (e.g., [2, 3]).

Psychological developmental assessment and healthrelated quality of life (HrQoL) measurement are recommended in several guidelines for the professional care of different IMDs (e.g., [4-8]). The term HrQoL reflects an individual's or a group's perceived impact of the disease and its treatment on their physical and mental health over time. Questionnaires measuring HrQoL provide a powerful tool to evaluate psychosocial stressors in the patients' and their families' lives [9]. Knowledge about HrQoL in children with IMDs is increasing, but findings are inconsistent. Previous studies have mainly investigated subgroups of these patients and the influence of treatment protocols on their HrQoL (e.g., [2, 10-14]).

In a recent study, Cano et al. found normal self-reported HrQoL in a large cohort of children suffering from intoxication-type IMDs [11]. In contrast, Bösch et al. described a significantly lower self-reported HrQoL in pediatric patients with IMDs of the intoxication type than in healthy children [12]. Interestingly, they found that the emotional scale score of patients with phenylketonuria (PKU) was lower compared to patients with IMDs causing acute exacerbations and life-threatening metabolic crises. This finding highlights the necessity to consider non-medical factors modulating HrQoL in these patients. However, studies on psychosocial influences on HrQoL of patients with IMDs are scarce [15]. Jamiolkowski et al. reported no correlation between HrQoL and behavioral or emotional problems in patients with organic acidurias and urea cycle disorders [16].

Our study investigated the HrQoL of pediatric patients with diverse IMDs receiving treatment at a pediatric metabolic center and aimed to understand associations between the patients' medical and psychosocial characteristics and their self-reported HrQoL to identify risk factors for impaired HrQoL. The findings could improve the psychosocial support for these patients and their families by economizing resources and individualizing support.

\section{Methods}

\section{Data collection}

This single-center retrospective study was conducted at the center of inherited metabolic diseases in Vienna, Austria, after ethical approval (Ethics Committee of the Medical University of Vienna, Number 1474/2018). Data was collected between 2002 and 2018 as part of the routine examination of children and adolescents with IMDs. The standardized routine examination protocol for these patients intends to provide regular psychological assessments at certain ages and defined time points, independent of clinical symptoms. It contains a specific set of questionnaires and psychological tests [17]. The results of the assessment are documented in the patient's medical record. For this study, we extracted the data from the medical records and included data of children from the age of seven.

\section{Participants}

The study sample consists of children and adolescents with a confirmed diagnosis of an IMD who are receiving or received treatment at the pediatric center of inherited metabolic diseases in Vienna and participated in the routine psychological assessment between 2002 and 2018. We excluded patients who did not complete the HrQoL questionnaire.

\section{Measures}

\section{HrQoL}

To evaluate the generic HrQoL, the German version of the standardized questionnaire KINDL-R [18] was administered. The questionnaire is available in several languages, and its psychometric properties have been tested in various studies (e.g., [19]). It contains age-appropriate versions for the patient's self-report and parent proxy reports. We evaluated data from the self-report versions KINDL Kid (7-12 years) and KINDL Kiddo (13-17 years). Each version consists of 24 items yielding a total HrQoL score and six subscales: physical well-being, emotional well-being, selfesteem, family, friends, and school. Higher scores represent better HrQoL. The maximum score is 100 for all subscales and the total score.

\section{Demographic data and diagnosis}

The patients' demographic and medical data were directly gathered from their medical files. Diagnoses were assigned to the diagnostic categories according to the SSIEM 
classification [1]. Infrequent diagnosis groups with less than ten patients were combined into a sixth category, "others."

\section{Expert rating on patient's health impairment}

To classify the patient's impairment in daily life due to the disease or its treatment, we established the new factor "health impairment." Three physicians who are experts in IMDs and were involved in treating the patients independently assigned the patients to one of three categories according to their impairment: (1) no impairment, (2) mild-moderate impairment in daily life (e.g., receiving protein-restricted diet, daily oral medication), and (3) severe impairment in everyday life (complex treatment, frequent stays in hospital, reduced life expectancy). In 90 patients $(44.1 \%)$, the rating of at least two experts matched. These patients were subsequently allocated to the respective impairment grade by the first author, who did not know the patients. Ninety-four patients $(46.1 \%)$ were known to only one physician and could not be assigned to a category.

\section{Intellectual functioning}

Assessment of intellectual functioning was performed with the Kaufman Assessment Battery for Children (K-ABC or K-ABC II, German version [20, 21]) or the Adaptive Intelligence Diagnosticum 2 (AID2 [22]). For this study, only the respective global test score (mental processing index for K-ABC/K-ABC II and IQ for AID 2) was used. It was divided into four categories: (a) above average: $>115$, (b) average: $85-115$, (c) below average: $70-84$, (d) cognitive impairment: $<70$.

\section{Psychosocial functioning}

To screen for emotional and behavioral problems, parents and patients aged $\geq 11$ years filled in the German version of the Strengths and Difficulties Questionnaire (SDQ) [23]. Both the self-report version (11-17 years) and the parent proxy version (4-17 years) of this behavioral screening questionnaire consist of 25 items divided between 5 subscales. Scores can be classified as normal, borderline, or abnormal. For this study, the total score was used. Parent proxy and self-report (if applicable) total scores in the normal range indicated a patient with no psychosocial functioning issues. Self-report, parent proxy, or both total scores above the average indicated "abnormal" functioning.

\section{Data analysis}

Descriptive statistics and ANOVAs were performed with the software SPSS (IBM SPSS Statistics 25.0). To evaluate the patients' self-reported HrQoL, we compared an age-matched group (11-17 years, $n=102$ ) of our sample to the published normative data from a large epidemiological study from Germany (Bella Study [9]). The Student $t$-test was used for the statistical comparison.

For each of the following variables, ANOVAs were performed to explore their influence on total $\mathrm{HrQoL}$ and subscale scores: (a) age group (two groups: 7-12 years vs. 13-17 years) and gender (two groups: female/male), (b) diagnostic category (six groups: amino acid and peptide metabolism disorders, carbohydrate metabolism disorders, fatty acid and ketone body metabolism defects, lysosomal disorders, disorders of vitamins and (non-protein) cofactors, others), (c) health impairment (three groups: none or almost none, mild to moderate, severe impairment), (d) intellectual functioning (four groups: above average, average, below average, cognitive impairment). For all analyses, HrQoL subscale scores and the HrQoL total score were entered as dependent variables. For subscale analyses, significance levels were calculated by applying Bonferroni-Holm correction to the $p$-values. Furthermore, we used the Bonferroni-Holm correction for the post hoc analyses of significant interaction effects. We applied the Student $t$-test to explore the influence of (e) family structure (two groups: living together with both parents versus other) and (f) psychosocial functioning (total SDQ score, two groups: normal versus abnormal). $P$ values $\leq 0.05$ were considered statistically significant. Effect sizes were calculated for significant results of post hoc analyses (Cohen's $d$ ), $t$-tests (Cohen's $d$ ), and ANOVA (partial eta squared, $\eta_{\text {Part }}^{2}$ ). Generally, Cohen's $d$ effect sizes $>0.2$ are considered small effects, $>0.5$ medium effects, and $>0.8$ large effects. Accordingly, for partial eta squared, Cohen suggested borders for small $\left(\eta^{2} \geq 0.01\right)$, medium $\left(\eta^{2} \geq 0.06\right)$, and large $\left(\eta^{2} \geq 0.14\right)$ effect sizes [24].

\section{Results}

\section{Sample characteristics}

In 235 out of 249 (94.3\%) psychological assessments, the child or adolescent filled in a KINDL questionnaire. From 31 patients, two datasets were available, as they had been assessed twice, once in childhood and once in adolescence. To avoid the bias of repeated measures, we chose only one of those datasets for evaluation. We alternated in including either the first or second assessment data. Hence, we 
analyzed data sets from 204 patients (118 children, 86 adolescents). Descriptive data on the study sample are summarized in Table 1.

\section{Comparison to normative data}

The mean KINDL-R total score of the sample was 74.1 \pm 11.2 . A $t$-test revealed no statistical difference in total HrQoL between the normative sample (total $\mathrm{HrQoL}=73.0 \pm 10.2$ ) and our age-matched subgroup of patients (total $\mathrm{HrQoL}=74.0 \pm 11.2$; Fig. 1). Our patients, however, reached higher HrQoL scores in the subscale "self-esteem" $(63.8 \pm 19.3)$ compared to the

Table 1 Patients' characteristics $(N=204)$

\begin{tabular}{|c|c|}
\hline \multicolumn{2}{|l|}{ Age at assessment } \\
\hline Mean & 11.89 years \\
\hline Standard deviation & 2.34 years \\
\hline Range & $7.00-17.33$ years \\
\hline Age group & $\begin{array}{l}\text { Total number } \\
\text { of patients (in } \\
\text { percentage) }\end{array}$ \\
\hline Children ( $7-12$ years) & $118(57.8 \%)$ \\
\hline Adolescents (13-17 years) & $86(42.2 \%)$ \\
\hline \multicolumn{2}{|l|}{ Gender } \\
\hline Male & $109(53.4 \%)$ \\
\hline Female & $95(46.6 \%)$ \\
\hline \multicolumn{2}{|l|}{ Family type } \\
\hline Both parents & $167(81.9 \%)$ \\
\hline Other & $37(18.1 \%)$ \\
\hline \multicolumn{2}{|l|}{ Diagnostic category } \\
\hline Amino acid and peptide metabolism disorders & $117(57.4 \%)$ \\
\hline Carbohydrate metabolism disorders & $35(17.2 \%)$ \\
\hline Fatty acid and ketone body metabolism defects & $12(5.9 \%)$ \\
\hline Lysosomal disorders & $19(9.3 \%)$ \\
\hline Disorders of vitamins and (non-protein) cofactors & $15(7.4 \%)$ \\
\hline Others & $6(2.9 \%)$ \\
\hline \multicolumn{2}{|c|}{ Health impairment (Patient's impairment in daily life) } \\
\hline None & $16(7.80 \%)$ \\
\hline Mild/moderate & $50(24.5 \%)$ \\
\hline Severe & $24(11.8 \%)$ \\
\hline No confirmed rating & $114(56.0 \%)$ \\
\hline \multicolumn{2}{|l|}{ Intellectual functioning (IQ) } \\
\hline Above average $(\geq 115)$ & $21(10.3 \%)$ \\
\hline Average $(85-114)$ & $118(57.8 \%)$ \\
\hline Below average $(70-84)$ & $41(20.1 \%)$ \\
\hline Disorder of intellectual development $(\leq 69)$ & $18(8.8 \%)$ \\
\hline No assessment & $6(2.9 \%)$ \\
\hline \multicolumn{2}{|l|}{ Psychosocial adjustment } \\
\hline Normal & $155(76.0 \%)$ \\
\hline Abnormal & $44(21.6 \%)$ \\
\hline Unknown & $5(2.5 \%)$ \\
\hline
\end{tabular}

Patients' mean age and frequency distributions of the assessed factors normative sample $($ score $=58.4 \pm 18.3, t(1994)=2.88, p=0.02$, Cohen's $d=0.29$; Fig. 1).

\section{Associations between psychosocial and medical characteristics of the patients and HrQoL}

Table 2 shows significant results of the statistical analyses on HrQoL modulating factors.

\section{Age and gender}

Analysis of the distribution of male and female patients did not reveal any significant difference $\left(X^{2}(2)=0.70, p=0.40\right)$. The two-way ANOVA showed a significant main effect of "age group" on the subscale "family" with a higher score in adolescents $(86.7 \pm 14.1)$ compared to children $(79.1 \pm 17.8$, $\left.F(1)=9.95, p=0.01, \eta_{\text {Part }}^{2}=0.05\right)$. We did not find any main effects or interaction effects on the total HrQoL score or the remaining subscales.

\section{Family structure}

We did not find any differences in HrQoL between children who live with their parents and children from single-parent families, stepfamilies, or foster families (family structure "other").

\section{Diagnostic category and health impairment}

The ANOVA with the factor "diagnostic category" revealed no significant differences in $\mathrm{HrQoL}$ between the various diagnostic categories. The ANOVA with the factor "health impairment" revealed significant main effects on the total HrQoL score $(F(2)=5.70, p=0.01)$ and the subscales "physical well-being" $(F(2)=9.90, p<0.001]$ and "family" $(F(2)=6.98, p=0.01)$. Post hoc results for the total HrQoL score confirmed a significantly lower score in patients with severe impairment (total HrQoL score $=66.8 \pm 12.2$ ) compared to patients with mild to moderate impairment (total HrQoL score $=76.1 \pm 11.3, p=0.01$, Cohen's $d=0.79$ ). Moreover, post hoc tests showed that patients with severe impairment scored significantly lower on the subscale "physical well-being" $(60.7 \pm 18.0)$ compared to patients with mild to moderate impairment (score $=79.2 \pm 15.6$, $p<0.001$, Cohen's $d=1.10$ ) and patients with no impairment (score $=74.6 \pm 18.2, p=0.04$, Cohen's $d=0.77$ ). As for the subscale "family," patients with mild to moderate impairment showed significantly higher scores $(85.1 \pm 13.2)$ than patients with no impairment (score $=68.0 \pm 21.9, p=0.02$, Cohen's $d=0.95$ ) and patients with severe impairment (score $=74.0 \pm 22.7, p=0.02$, Cohen's $d=0.70$ ). A chisquare test ruled out distributional effects between age-group and impairment grade $\left(X^{2}(1)=0.09, p=0.96\right)$. 
Fig. 1 Comparison of the agematched subgroup of patients with German normative data (mean subscale-scores, standard deviation, and total HrQoL score). The significant group difference is marked with $p$-value and Cohen's $d$

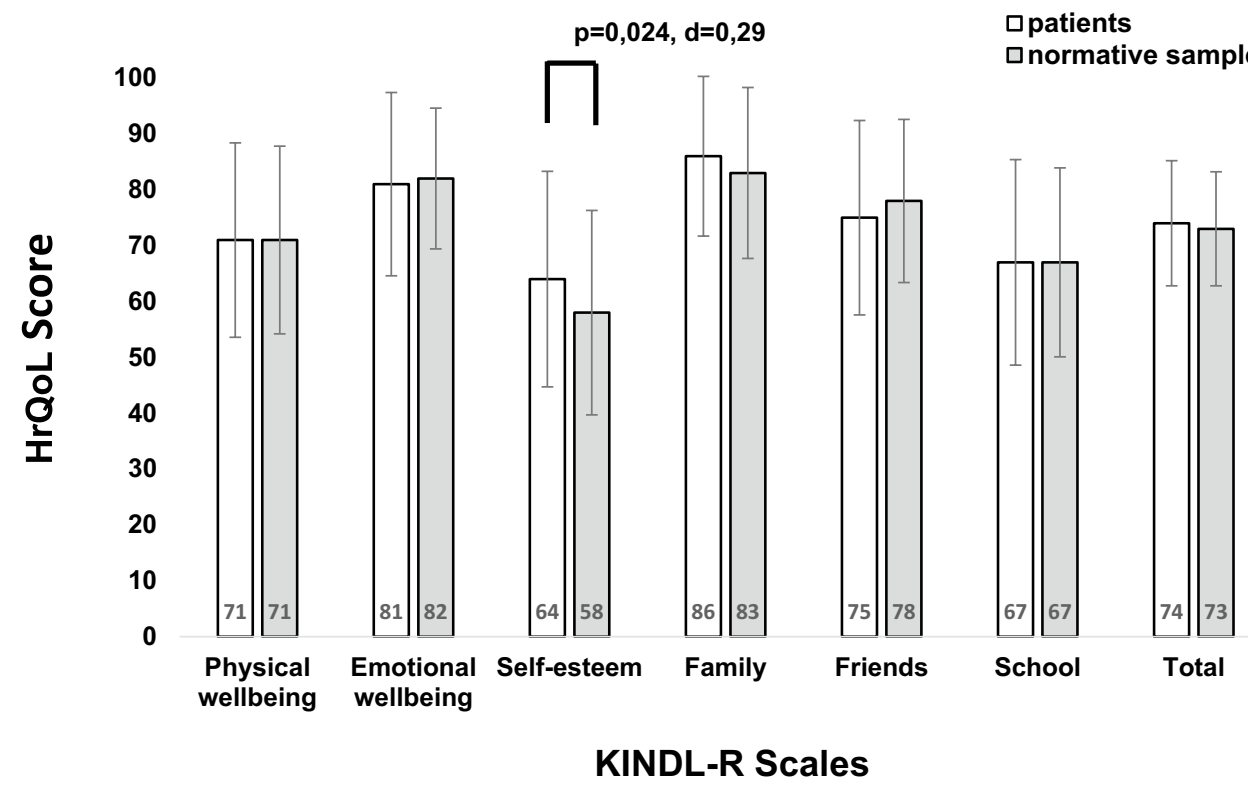

\section{Intellectual functioning}

ANOVA on HrQoL with the factor "intellectual functioning" (four groups) indicated a significant influence $(F(3)=3.66, p=0.01)$ on the total HrQoL. Post hoc testing confirmed lower total HrQoL scores in patients with disorders of intellectual development (total $\mathrm{HrQoL}=66.9 \pm 8.5$ ) compared to patients with average intellectual abilities (total HrQoL $=75.8 \pm 10.9, p=0.004$, Cohen's $d=0.84$ ). Moreover, ANOVA revealed a significant main effect of the factor "intellectual functioning" on the subscale "physical well-being" $(F(3)=4.48, p=0.03)$. Post hoc analysis revealed lower "physical well-being" scores in patients with disorders of intellectual development $($ score $=60.4 \pm 13.7$ ) compared to patients with average intellectual abilities (score $=75.1 \pm 17.3, p=0.003$, Cohen's $d=0.87$ ) and patients with higher-than-average intellectual abilities (score $=77.6 \pm 12.8, p<0.001$, Cohen's $d=1.31$ ). A chisquare test ruled out distributional effects of intellectual functioning and impairment grade $\left(X^{2}(6)=6.91, p=0.33\right)$.

Table 2 Overview of subscales with significant group differences in HrQoL scores

\begin{tabular}{|c|c|c|c|c|c|c|c|c|}
\hline HrQoL scale & & $\begin{array}{l}\text { Physical } \\
\text { well- } \\
\text { being } \\
\text { Mean }\end{array}$ & $\begin{array}{l}\text { Emotional } \\
\text { well-being } \\
\text { Mean }\end{array}$ & $\begin{array}{l}\text { Self-esteem } \\
\text { Mean }\end{array}$ & $\begin{array}{l}\text { Family } \\
\text { Mean }\end{array}$ & $\begin{array}{l}\text { Friends } \\
\text { Mean }\end{array}$ & $\begin{array}{l}\text { School } \\
\text { Mean }\end{array}$ & $\begin{array}{l}\text { Total HrQoL } \\
\text { Mean }\end{array}$ \\
\hline Total $(N=204)$ & & 72.9 & 80.8 & 63.6 & 82.3 & 75.9 & 68.9 & 74.1 \\
\hline \multirow[t]{2}{*}{ Age groups } & Children ( $7-12$ years) & & & & 79.1 & & & \\
\hline & Adolescents (13-17 years) & & & & 86.7 & & & \\
\hline \multirow{3}{*}{$\begin{array}{l}\text { Health impairment (expert } \\
\text { rating) }\end{array}$} & No impairment & 74.6 & & & 68.0 & & & \\
\hline & Mild-moderate & 79.2 & & & 85.1 & & & 76.1 \\
\hline & Severe & 60.7 & & & 74.0 & & & 66.8 \\
\hline \multirow[t]{4}{*}{ Intellectual functioning } & Above average & 77.6 & & & & & & \\
\hline & Average & 75.1 & & & & & & 75.8 \\
\hline & Below average & & & & & & & \\
\hline & $\begin{array}{l}\text { Disorder of intellectual } \\
\text { development }\end{array}$ & 60.4 & & & & & & 66.9 \\
\hline \multirow[t]{2}{*}{ Psychosocial functioning } & Normal & 75.4 & 84.1 & & 85.3 & 78.6 & 71.8 & 76.6 \\
\hline & Abnormal & 63.7 & 70.9 & & 72.6 & 67.3 & 59.2 & 65.8 \\
\hline
\end{tabular}

HrQoL modulating factors. Significant hrQoL scores as per ANOVA and post hoc tests with $p$-values $<0.05$ are highlighted: bold, higher HrQoL scores; italic, lower HrQoL scores. Missing values represent non-significant results 


\section{Psychosocial functioning}

SDQ self-report and parent proxy-report data of 199 patients were collected. In 155 patients (76\%), the total difficulties score was normal. Patients with an abnormal total difficulties score showed significantly lower total HrQoL scores $(65.8 \pm 10.1)$ compared to patients with a normal total difficulties score (total HrQoL score $=76.6 \pm 10.2$, $t(196)=6.16, p<0.001$, Cohen's $d=1.08)$. HrQoL scores of patients with an abnormal total difficulty score remained consistently lower in all subscales (except "self-esteem") with all $p$-values below 0.01 .

\section{Discussion}

In this study, we investigated the self-reported generic HrQoL and its associations with psychosocial and medical characteristics in a cohort of over 200 children and adolescents affected by an IMD and receiving special care in a large, acknowledged center for pediatric IMDs. Interestingly, our adolescent patients' HrQoL was comparable to a normative sample from a German epidemiological study [9]. This finding is in line with a recent report on normal self-reported HrQoL in a subgroup of French pediatric IMD patients [11].

Moreover, these results are compatible with several HrQoL studies in children with congenital or early infancy chronic diseases showing a normal self-reported HrQoL [25]. It is assumed that a normal HrQoL indicates an adaptation to the burden of disease and the development of coping strategies, especially in non-progressive conditions [25, 26]. Thimm et al. assumed that living with an IMD might entail valuable aspects such as more supportive parental care or feelings of satisfaction about managing treatment requirements [27]. Interestingly, we found even higher HrQoL scores for the subscale "self-esteem" in our cohort, corroborating this assumption. Importantly, Cano et al. who used a French questionnaire and French normative data, report higher than average HrQoL scores in the older subgroup of patients (13-17 years) and lower HrQoL scores in the younger subgroup of children (aged 8-12 years) [11].

In contrast to our results, Bösch et al. who adopted the PedsQL to measure HrQoL in a slightly younger cohort (average age 11 years), found significantly lower scores compared to normative data from healthy American children [12]. Since our comparison was limited to patients aged 11-17 (average age 14 years), findings on average and above-average HrQoL scores might apply to older children and adolescents who have already developed coping strategies and learned how to co-manage their treatments. However, our finding of normal HrQoL in adolescents with IMDs should be interpreted with caution due to the diseases' heterogeneity. It is probably not applicable to adolescents with severe disease-related burdens. Furthermore, additional analyses of our dataset revealed that the result of a higher self-esteem in the adolescent patient group is especially true for patients with normal cognitive and psychosocial functioning (data not shown).

Notably, scores of the subscale "family" were lower in children than in adolescents. Cano et al. reported similar findings in children suffering from intoxication type IMDs [11]. Adolescents might become aware that they depend more on their caregivers than healthy peers and avoid conflicts within the household. Moreover, as adolescents were conscious that the questionnaire was not anonymous, the social-desirability bias could have interfered with the interpretation of HrQoL scores.

Several studies on chronically ill and healthy children showed that gender influences HrQoL [9, 28]. Interestingly, we did not find gender effects in our data, suggesting that other factors have a more robust influence on our cohort.

The diagnostic category itself did not significantly modulate the HrQoL. This finding may be due to the variability within diagnostic categories regarding the influence of the various diseases and treatments on the patients' daily lives. The health impairment grade, however, influenced the selfreported HrQoL of our patients. A higher grade of health impairment was associated with a lower HrQoL, especially on the subscales "physical well-being" and "family." We assume that significant disease-related impairments impose challenges and limitations that protective factors cannot mitigate. Furthermore, Bilginsoy et al. showed that controlling the effects of the child's illness on their social life represents a source of stress for parents of children suffering from PKU [29]. Hence, the perception of parental stress might have influenced the family HrQoL of our patients.

On the other hand, patients without health impairment also showed lower HrQoL scores on the subscale "family" than mildly impaired patients. This finding supports the assumption that children without impairment tend to have more conflicts with their parents and underline the importance of the family system for patients bearing the burden of their diagnosis. Hence, evaluating the patient's health impairment helps identify patients and families needing enhanced psychosocial support. However, to best support the children and their families, it would be important to also consider the parents' proxy reports as well as the parental HrQoL.

Furthermore, we found a significant association between HrQoL and intellectual functioning; patients with disorders of intellectual development showed lower total HrQoL and physical well-being scores than children with average or higher intellectual abilities. As intellectual functioning levels were equally distributed among health impairment categories, a higher impairment grade in patients with disorders of intellectual development does not explain this result. We assume that lower cognitive capacities prevent patients from 
developing optimal strategies to cope with the limitations resulting from their disease thus indicating the need for support in establishing adequate coping strategies. As expected, patients with lower psychosocial functioning reported a lower HrQoL in almost all KINDL scales. Similar findings were reported for children with propionic academia [2], chronically ill children in general [28], and healthy children [9]. In a longitudinal study, Otto et al. showed that mental health problems are a risk factor for diminished $\mathrm{HrQoL}$ in children and should be addressed in prevention programs [30].

\section{Limitations}

Although large, our sample reflects the frequency distribution of different IMDs in our metabolic center and may not be representative. Disease-specific effects of more frequent diagnostic groups of IMDs might be overrepresented. Moreover, the KINDL-R is a generic questionnaire that is less sensitive to disease- or treatment-specific limitations of $\mathrm{HrQoL}$ than disease-specific questionnaires [16]. Applying diseasespecific HrQoL questionnaires could have possibly revealed different findings. Furthermore, comparison with normative data was restricted to children older than ten years. Although we excluded patients with evident and severe difficulties in verbal comprehension, patients with impaired cognitive functioning might have had difficulties in understanding and answering the KINDL questionnaire. Such difficulties could have had an influence on their HrQoL scores. Furthermore, the patients knew that the results of their psychological assessments would be discussed with their parents. Thus, social desirability might have inflated scores.

\section{Conclusion}

We found that, on average, adolescents suffering from IMDs can achieve a normal HrQoL. Our study revealed three essential factors that affect the self-reported HrQoL of pediatric IMD patients highlighting the need for distinct psychological support for specific subgroups.

(I) Health impairment: The care team needs to judge the impairment due to the disease or treatment and offer psychological support to highly impaired patients. As family $\mathrm{HrQoL}$ is substantially affected by health impairment, family counseling and therapy are recommended.

(II) Cognitive functioning: Patients with disorders of intellectual development should be identified and offered support in establishing coping strategies.

(III) Psychosocial functioning: Screening for mental health problems in IMD patients with a brief questionnaire addressing behavioral and emotional problems is essential. Early treatment of mental health problems could positively impact the patients' HrQoL.

Acknowledgements We acknowledge the support of the Scientific Publishing Service provided by P. Voitl and E. Tomasco, on behalf of the Austrian Pediatric Society.

Authors' contributions All authors substantially contributed to the work and were involved in (a) conception and design of the study and/ or analysis and interpretation of data and (b) revising the article critically for important intellectual content. Marion Herle conceptualized the study and collected the data. Sandy Siegert and Marion Herle analyzed the data, wrote the first version of the manuscript, drafted, and wrote the final version of the manuscript. Anne Roscher, Dorothea Möslinger, and Vassiliki Konstantopoulou provided their clinical expert knowledge and critically reviewed the later versions of the manuscript. All authors approved the final manuscript as submitted and agree to be accountable for all aspects of the work. All authors confirm the absence of previous similar publications. All authors confirm that this manuscript has not been submitted to another journal.

Funding Open access funding provided by Medical University of Vienna.

Availability of data and material (data transparency) All data are available on request.

Code availability N/A.

\section{Declarations}

Ethics approval This research study was conducted retrospectively from data obtained for clinical purposes. It was approved by the Ethics Committee of the Medical University of Vienna (EK-number: 1474/2018).

\section{Consent to participate N/A}

Consent for publication N/A

Conflict of interest The authors declare no competing interests.

Open Access This article is licensed under a Creative Commons Attribution 4.0 International License, which permits use, sharing, adaptation, distribution and reproduction in any medium or format, as long as you give appropriate credit to the original author(s) and the source, provide a link to the Creative Commons licence, and indicate if changes were made. The images or other third party material in this article are included in the article's Creative Commons licence, unless indicated otherwise in a credit line to the material. If material is not included in the article's Creative Commons licence and your intended use is not permitted by statutory regulation or exceeds the permitted use, you will need to obtain permission directly from the copyright holder. To view a copy of this licence, visit http://creativecommons.org/licenses/by/4.0/. 


\section{References}

1. Ferreira CR, Rahman S, Keller M, Zschocke J (2021) ICIMD Advisory Group. An international classification of inherited metabolic disorders (ICIMD). J Inherit Metab Dis 44(1):164-177. https://doi.org/10.1002/jimd.12348. PMID: 33340416

2. Grünert SC, Müllerleile S, De Silva L et al (2013) Propionic acidemia: clinical course and outcome in 55 pediatric and adolescent patients. Orphanet J Rare Dis 8:6

3. Batshaw ML, Tuchman M, Summar M, Seminara J (2014) Members of the Urea Cycle Disorders Consortium. A longitudinal study of urea cycle disorders. Mol Genet Metab 113(1-2):127-130

4. Boy N, Mühlhausen C, Maier EM et al (2017) Proposed recommendations for diagnosing and managing individuals with glutaric aciduria type I: second revision. J Inherit Metab Dis 40(1):75-101

5. Welling L, Bernstein LE, Berry GT et al (2017) International clinical guideline for the management of classical galactosemia: diagnosis, treatment, and follow-up. J Inherit Metab Dis 40(2):171-176

6. Häberle J, Burlina A, Chakrapani A et al (2019) Suggested guidelines for the diagnosis and management of urea cycle disorders: First revision. J Inherit Metab Dis 42(6):1192-1230

7. Vockley J, Andersson HC, Antshel KM et al (2014) Phenylalanine hydroxylase deficiency: diagnosis and management guideline [published correction appears in Genet Med 16(4):356]. Genet Med 16(2):188-200

8. Baumgartner MR, Hörster F, Dionisi-Vici C et al (2014) Proposed guidelines for the diagnosis and management of methylmalonic and propionic acidemia. Orphanet J Rare Dis 9:130

9. Ravens-Sieberer U, Erhart M, Wille N et al (2008) Health-related quality of life in children and adolescents in Germany: results of the BELLA study. Eur Child Adolesc Psychiatry 17:148-156

10. Ten Hoedt AE, Maurice-Stam H, Boelen CC et al (2011) Parenting a child with phenylketonuria or galactosemia: implications for health-related Quality of life. J Inherit Metab Dis 34(2):391-398

11. Cano A, Resseguier N, Ouattara A et al (2020) Health status of French young patients with inborn errors of metabolism with lifelong restricted diet. J Pediatr 220:184-192.e6

12. Bösch F, Landolt MA, Baumgartner MR et al (2020) Healthrelated quality of life in paediatric patients with intoxication-type inborn errors of metabolism: analysis of an international data set. J Inherit Metab Dis. https://doi.org/10.1002/jIEM.12301

13. Eminoglu TF, Soysal SA, Tumer L, Okur I, Hasanoglu A (2013) Quality of life in children treated with restrictive diet for inherited metabolic disease. Pediatr Int 55(4):428-433

14. Bosch AM, Burlina A, Cunningham A et al (2015) Assessment of the impact of phenylketonuria and its treatment on quality of life of patients and parents from seven European countries. Orphanet J Rare Dis 10:80

15. Zeltner NA, Huemer M, Baumgartner MR, Landolt MA (2014) Quality of life, psychological adjustment, and adaptive functioning of patients with intoxication-type inborn errors of metabolism - a systematic review. Orphanet J Rare Dis 9:159

16. Jamiolkowski D, Kölker S, Glahn EM et al (2016) Behavioural and emotional problems, intellectual impairment and health-related quality of life in patients with organic acidurias and urea cycle disorders. J Inherit Metab Dis 39(2):231-241

17. Herle M, Möslinger D, Zeyda M, Greber-Platzer S, Konstantopoulou V (2018) Psychological follow-up of patients with inborn errors of metabolism diagnosed in newborn screening at the Department of Pediatrics and Adolescent Medicine, Medical University of Vienna. 25th Annual Meeting of the German Society of Newborn Screening. Int J Neonatal Screen 4(2): 17

18. Ravens-Sieberer U (2003) Der KINDL-R Fragebogen zur Erfassung der gesundheitsbezogenen Lebensqualität bei Kindern und Jugendlichen—Revidierte Form. In: Schumacher JKA, Brähler E (eds) Diagnostische Verfahren zu Lebensqualität und Wohlbefinden. Hogrefe, Göttingen, pp 184-188

19. Bullinger M, Brütt AL, Erhart M, Ravens-Sieberer U (2008) BELLA Study Group. Psychometric properties of the KINDL-R questionnaire: results of the BELLA study. Eur Child Adolesc Psychiatry 17 Suppl 1:125-32

20. Melchers P, Preuss U (eds.) (2003) Kaufman-Assessment Battery for Children. German version (K-ABC) (6. Edition) Frankfurt. Swets \& Zeitlinger

21. Melchers P, Melchers M (2015) Kaufman-Assessment Battery for Children - second edition (K-ABC II). German version Frankfurt. Pearson Assessment

22. Kubinger KD, Wurst E (2000) Adaptives Intelligenz-Diagnostikum 2 (AID-2). Göttingen. Beltz

23. Goodman R (1997) The Strengths and Difficulties Questionnaire: a research note. J Child Psychol Psychiatry 38(5):581-586

24. Cohen J (1988) Statistical power analysis for the behavioral sciences (2nd ed.). Hillsdale, NJ: Lawrence Erlbaum Associates

25. Jardine J, Glinianaia SV, McConachie H, Embleton ND, Rankin J (2014) Self-reported quality of life of young children with conditions from early infancy: a systematic review. Pediatrics 134(4):e1129-e1148

26. Landolt MA, Nuoffer JM, Steinmann B, Superti-Furga A (2002) Quality of life and psychologic adjustment in children and adolescents with early treated phenylketonuria can be normal. J Pediatr 140(5):516-521

27. Thimm E, Schmidt LE, Heldt K et al (2013) Health-related quality of life in children and adolescents with phenylketonuria: unimpaired $\mathrm{HrQoL}$ in patients but feared school failure in parents. $\mathrm{J}$ Inherit Metab Dis 36:767-772

28. Barthel D, Ravens-Sieberer U, Nolte S, Thyen U, Klein M, Walter O, Meyrose AK, Rose M, Otto C (2018) Predictors of healthrelated quality of life in chronically ill children and adolescents over time. J Psychosom Res 109:63-70

29. Bilginsoy C, Waitzman N, Leonard CO, Ernst SL (2005) Living with phenylketonuria: perspectives of patients and their families. J Inherit Metab Dis 28(5):639-649

30. Otto C, Haller AC, Klasen F, Hölling H, Bullinger M, RavensSieberer U (2017) BELLA study group. Risk and protective factors of health-related quality of life in children and adolescents: results of the longitudinal BELLA study. PLoS One 12(12):e0190363

Publisher's Note Springer Nature remains neutral with regard to jurisdictional claims in published maps and institutional affiliations. 